\title{
"Rechtliche Verbindlichkeit» der Richtlinien zur Gewinnung von Blut und Blutbestandteilen und zur Anwendung von Blutprodukten (Hämotherapie) nach der Novellierung des Transfusionsgesetzes
}

\author{
Horst Hasskarl Bita Bakhschai \\ Harms Hasskarl Melzer Rechtsanwälte, Ludwigshafen, Deutschland
}

\section{Schlüsselwörter}

Richtlinien zur Gewinnung von Blut und Blutbestandteilen und zur Anwendung von Blutprodukten (Hämotherapie) der Bundesärztekammer · Rechtsgrundlage

\section{Zusammenfassung}

Vor Inkrafttreten des Gewebegesetzes stellte die Bundesärztekammer aufgrund des $\S 12$ Transfusionsgesetz (TFG) in Richtlinien den allgemein anerkannten Stand der medizinischen Wissenschaft und Technik unter anderem zur Gewinnung von Blut und Blutbestandteilen fest. Durch das Gewebegesetz fand diesbezüglich eine Neuerung statt. Anstelle des bisherigen § 12 TFG wurde ein inhaltlich neuer $\S 12$ TFG geschaffen. Dieser enthält eine Ermächtigung zum Erlass einer Rechtsverordnung durch das Bundesministerium für Gesundheit. Außerdem wurde ein neuer $\S 12 a$ TFG geschaffen. Dieser enthält als Ausgleich für die ursprünglich in § 12 TFG alte Fassung geregelte umfassende Richtlinienkompetenz der Bundesärztekammer eine nunmehr deutlich begrenzte Ermächtigung der Bundesärztekammer zur lediglich ergänzenden Feststellung des Stands der Erkenntnisse der medizinischen Wissenschaft und Technik zur Gewinnung von Blut und Blutbestandteilen. Im Juli 2010 wurden - auf der Grundlage der §§ 12a und 18 TFG - von der Bundesärztekammer im Einvernehmen mit dem Paul-Ehrlich-Institut die «Richtlinien zur Gewinnung von Blut und Blutbestandteilen und zur Anwendung von Blutprodukten (Hämotherapie)" (nachfolgend BlutRiLi) in neuester Fassung im Bundesanzeiger bekannt gemacht. Es bestehen rechtliche Zweifel, ob die BlutRiLi, soweit sie die Gewinnung von Blut und Blutbestandteilen betreffen, in rechtmäßiger Weise gestützt auf § 12a TFG erlassen werden konnten.

\section{Keywords}

Guidelines Regarding the Collection of Blood and Blood Components and the Application of Blood Products (Hemotherapy) of the Federal Chamber of Physicians · Legal basis

\section{Summary}

'Legally Binding Force' of Guidelines Regarding the Collection of Blood and Blood Components and the Application of Blood Products (Hemotherapy) after Amendment of the German Transfusion Law

Before the Gewebegesetz (German Tissue Act) came into effect (on August 1, 2007), the Bundesärztekammer (German Federal Chamber of Physicians) was the institution which was legally entitled to publish the generally accepted standards of medicinal science and technology relating to the collection of blood and blood components. The legal basis was section 12 Transfusionsgeseztz (TFG; German Transfusion Act). Due to an amendment of the TFG by the Gewebegesetz the former section 12 TFG was replaced by a new section 12 TFG with a new content. This new section contains an authorization to the Bundesministerium für Gesundheit (BMG; German Federal Ministry of Health) to issue a regulation. Furthermore, a new section 12a TFG has been created. This section contains an obviously restricted authorization in favor of the Bundesärztekammer which is now only empowered to publish the standards of knowledge of medicinal science and technology relating to the collection of blood and blood components in addition to the provisions of the regulation of the BMG. In July 2010, legally based on sections 12a and 18 TFG the 'Richtlinien zur Gewinnung von Blut und Blutbestandteilen und zur Anwendung von Blutprodukten (Hämotherapie)' (Guidelines Regarding the Collection of Blood and Blood Components and the Application of Blood Products (Hemotherapy)) of the Bundesärztekammer have been published in agreement with the Paul-Ehrlich-Institut in the Bundesanzeiger (Federal Gazette). Hence there are legal doubts whether the 'Richtlinien zur Gewinnung von Blut und Blutbestandteilen und zur Anwendung von Blutprodukten (Hämotherapie)' could correctly be based on section 12a TFG as far as the collection of blood and blood components is concerned.

\begin{tabular}{ll}
\hline KARGER & @ 2011 S. Karger GmbH, Freiburg \\
Fax +497614520714 & Accessible online at: \\
Information@Karger.de & www.karger.com/tmh
\end{tabular}




\section{Einleitung}

Vor Inkrafttreten des Gewebegesetzes [1], wodurch unter anderem das Transfusionsgesetz (TFG) geändert wurde, stellte die Bundesärztekammer - im Einvernehmen mit der zuständigen Bundesoberbehörde und nach Anhörung von Sachverständigen unter Berücksichtigung der Richtlinien und Empfehlungen der Europäischen Union, des Europarates und der Weltgesundheitsorganisation zu Blut und Blutbestandteilen in Richtlinien den allgemein anerkannten Stand der medizinischen Wissenschaft und Technik unter anderem zur Gewinnung von Blut und Blutbestandteilen fest (vgl. § 12 Abs. 1 S. 1 TFG alte Fassung [2]). Die Bundesärztekammer hatte in diesem Bereich sozusagen - von gesetzlichen Vorschriften abgesehen - ein Monopol zur Feststellung - nicht Festlegung - des Standes der medizinischen Wissenschaft und Technik inne.

Durch das Gewebegesetz fand diesbezüglich eine Neuerung statt. Anstelle des bisherigen $\S 12$ TFG wurde ein inhaltlich neuer $\S 12$ TFG geschaffen. Dieser enthält eine Ermächtigung zum Erlass einer Rechtsverordnung durch das Bundesministerium für Gesundheit (BMG). Eine Rechtsverordnung ist neben einem Gesetz - unter anderem nämlich zur verbindlichen Umsetzung von EG(EU)-Recht das ausschließlich geeignete und erforderliche Mittel. (Insoweit unzutreffend und irreführend ist die allgemeine Anmerkung in den «Richtlinien zur Gewinnung von Blut und Blutbestandteilen und zur Anwendung von Blutprodukten (Hämotherapie)» (nachfolgend BlutRiLi) (Kapitel 1 Allgemeines) mit folgendem Wortlaut: «Diese Richtlinien setzen auch die einschlägigen Richtlinien und Empfehlungen der Europäischen Union um.». Zur Umsetzung von EG-Richtlinen im Blutbereich siehe [3]). Auf Empfehlung des Bundestagsausschusses für Gesundheit wurde in diesem Zusammenhang außerdem ein neuer $\$ 12$ a TFG geschaffen [4]. Dieser enthält als Ausgleich für die ursprünglich in $\S 12$ TFG alte Fassung geregelte weite Richtlinienkompetenz der Bundesärztekammer eine modifizierte und inhaltlich deutlich reduzierte Ermächtigung der Bundesärztekammer zur Feststellung des Stands der Erkenntnisse der medizinischen Wissenschaft und Technik zur Gewinnung von Blut und Blutbestandteilen.

Im Juli 2010 wurden - auf der Grundlage der $\S$ 12a (nach der amtlichen Bekanntmachung im Bundesanzeiger wird die BlutRiLi fälschlicherweise auf $\S 12$ TFG und nicht - wie zutreffend - auf § 12a TFG gestützt) und 18 TFG - von der Bundesärztekammer im Einvernehmen mit dem Paul-Ehrlich-Institut die BlutRiLi in neuester Fassung im Bundesanzeiger bekannt gemacht (Zweite Richtlinienanpassung 2010, BAnz. Nr. 101a vom 9. Juli 2010; abrufbar unter www.bundesaerztekammer.de). Die vorgenommenen Änderungen betreffen unter anderem die Beseitigung von Diskrepanzen zwischen Regelungen der BlutRiLi einerseits und andererseits den separaten QuerschnittsLeitlinien der Bundesärztekammer von 2008 zur Therapie mit Blutkomponenten und Plasmaderviaten (sowohl in deutscher als auch in englischer Sprache abrufbar unter www.bundesaerztekammer.de).
Die BlutRiLi gelten für alle Ärzte, die mit dem Gewinnen, Herstellen, Lagern, Abgeben oder Inverkehrbringen von Blut, Blutbestandteilen oder Blutprodukten, der Durchführung von blutgruppenserologischen und weiteren immunhämatologischen Untersuchungen sowie der Anwendung von Blutprodukten und der entsprechenden Nachsorge befasst sind (Kapitel 1.1 BlutRiLi). Nach Kapitel 1.2 BlutRiLi stellen diese Richtlinien gemäß $\S 12 \mathrm{a}$ und 18 TFG [5] den allgemein anerkannten Stand der medizinischen Wissenschaft und Technik zur Gewinnung von Blut und Blutbestandteilen und zur Anwendung von Blutprodukten fest.

Nachfolgend soll die Frage beleuchtet werden, ob die BlutRiLi bereits zum gegenwärtigen Zeitpunkt in rechtmäßiger Weise, gestützt auf $\S 12 \mathrm{a}$ TFG, erlassen werden konnten. Ist nicht zunächst der Erlass einer auf $\S 12$ TFG gestützten Rechtsverordnung durch das BMG erforderlich, damit die Bundesärztekammer erst dann und nur dann den allgemein anerkannten Stand der Erkenntnisse der medizinischen Wissenschaft und Technik zur Gewinnung von Blut und Blutbestandteilen ergänzend zu den Vorschriften der Rechtsverordnung nach $\S 12$ im Einvernehmen mit der zuständigen Bundesoberbehörde in Richtlinien feststellen kann? Oder wird etwa das BMG nur dann den Erlass einer Verordnung nach $\S 12$ TFG in Betracht ziehen, wenn sich in der Praxis herausstellen sollte, dass die bereits existierenden Regeln, zu denen auch die auf $\S 12$ a TFG gestützten BlutRiLi der Bundesärztekammer gehören, nicht ausreichen, nicht beachtet oder nicht dem aktuellen Stand der Erkenntnisse der medizinischen Wissenschaft und Technik angepasst werden?

\section{Ermächtigungsgrundlage des $\S 12$ TFG}

\section{Wortlaut und amtliche Begründung}

Die Ermächtigungsgrundlage des $§ 12$ TFG (allgemeine verfassungsrechtliche Anforderungen an eine Verordnungsermächtigungsnorm sind in Artikel 80 des Grundgesetzes geregelt) wurde durch Artikel 3 Ziffer 3 des Gewebegesetzes [1] mit nachstehendem Wortlaut in das TFG eingeführt:

«Das Bundesministerium für Gesundheit kann durch Rechtsverordnung mit Zustimmung des Bundesrates nach Anhörung der Bundesärztekammer und weiterer Sachverständiger die fachlichen Anforderungen nach diesem Abschnitt regeln, sofern dies zur Abwehr von Gefahren für die Gesundheit von Menschen oder zur Risikovorsorge erforderlich ist. In der Rechtsverordnung kann insbesondere das Nähere zu den Anforderungen an

1. die Spendeeinrichtungen,

2. die Auswahl und Untersuchung der spendenden Personen,

3. die Aufklärung und Einwilligung der spendenden Personen,

4. die Spendeentnahme, 
5. die Spenderimmunisierung und die Vorbehandlung zur Blutstammzellentnahme und

6. die Dokumentation der Spendeentnahme und den Schutz der dokumentierten Daten geregelt werden. Das Bundesministerium für Gesundheit kann die Ermächtigung nach Satz 1 durch Rechtsverordnung ohne Zustimmung des Bundesrates auf die zuständige Bundesoberbehörde übertragen.»

Die amtliche Begründung [6] zu dieser Vorschrift lautet:

«Die ursprüngliche Richtlinienkompetenz der Bundesärztekammer und der zuständigen Bundesoberbehörde zur alleinigen Feststellung des allgemein anerkannten Standes der medizinischen Wissenschaft und Technik wird durch eine Verordnungsermächtigung für das Bundesministerium für Gesundheit ersetzt. Eine Verordnung ist das geeignete Instrument zur verbindlichen Umsetzung von EG-Recht, das im Bereich der Qualität und Sicherheit von Blutprodukten einer ständigen Revision der fachlichen Anforderungen in Durchführungsrichtlinien der EU-Kommission unterworfen sein wird. Es handelt sich um Mindeststandards, die verbindlich in nationales Recht umgesetzt werden müssen. Die Verordnung kann darüber hinausgehend höhere Standards festlegen. Sofern die Fachkreise weitere oder konkretisierende Standards für erforderlich halten, können diese in Empfehlungen zur Konkretisierung des Standes der medizinischen Wissenschaft und Technik festgelegt werden. Wenn die Verordnungsermächtigung nicht ausschließlich zur Umsetzung von EG-Recht genutzt werden soll, ist dies nur zulässig, wenn es zur Abwehr von Gefahren für die Gesundheit von Menschen oder zur Risikovorsorge erforderlich ist. Insoweit bedarf es dann einer ausdrücklichen Begründung. Es werden die wichtigsten Themen für eine Verordnung genannt, die nicht abschließend sind. Das Bundesministerium für Gesundheit kann durch Rechtsverordnung (ohne Zustimmung des Bundesrates) die Befugnis zum Erlass der Verordnung auf die zuständige Bundesoberbehörde, das Paul-Ehrlich-Institut, delegieren, was dann angezeigt ist, wenn detaillierte fachliche Inhalte geregelt werden müssen. Es kann sich die Ermächtigung aber auch wieder zurückholen. Vor Erlass der Verordnung sind die Bundesärztekammer und weitere Sachverständige insbesondere aus dem Bereich der betroffenen Hersteller anzuhören.»

(Hervorhebungen durch die Verfasser)

\section{Zusammenhang mit Ermächtigungsnorm des § 30 Abs. 1 TFG}

Durch die bestehenden Ermächtigungsgrundlagen des TFG zum Erlass von Rechtsverordnungen für spezielle Sachbereiche (vgl. §§ 12, 20, 23 TFG), insbesondere durch $\S 12$ TFG, ist gesichert, dass in Verbindung mit der Ermächtigungsnorm des $\S 30$ Abs. 1 TFG nahezu alle künftigen Regelungsinhalte der
EG(EU)-Durchführungsrichtlinien [7-9] zur Richtlinie 2002/98/EG [10] mit einer Rechtsverordnung nach dem TFG umgesetzt werden könnten [11, § $30 \mathrm{Rdn} .1$ ]. Der Vorteil der Ermächtigung in $\S 30$ Abs. 1 TFG besteht darin, dass bestimmte Voraussetzungen der Spezialermächtigungen nicht zwingend erfüllt sein müssen. Das gilt bei $\S 12$ TFG z.B. für die Voraussetzungen, dass die Verordnung «zur Abwehr von Gefahren für die Gesundheit von Menschen oder zur Risikovorsorge erforderlich» sein muss. Diese einschränkende Voraussetzung zur Nutzung der Ermächtigung in §12 TFG wird durch die insoweit voraussetzungslose Ermächtigung in $\S 30$ Abs. 1 TFG ersetzt. Sie ist dann für den Erlass der Verordnung nach § 12 TFG kein Hindernis, obwohl auch die blutbezogenen Regelungen der EG-Richtlinien der Sicherheit und der Risikovorsorge dienen [11, § $30 \mathrm{Rdn} .3]$. Letzteres kann nicht nur den Erwägungsgründen der im Blutbereich ergangenen EG-Richtlinien entnommen werden, sondern auch Artikel 168 Abs. 4 lit. a AEUV [12], der wie folgt lautet:

«(...) tragen das Europäische Parlament und der Rat (...) mit folgenden Maßnahmen zur Verwirklichung der Ziele dieses Artikels bei, um den gemeinen Sicherheitsanliegen Rechnung zu tragen:

a) Maßnahmen zur Festlegung hoher Qualitäts- und Sicherheitsstandards für Organe und Substanzen menschlichen Ursprungs sowie für Blut und Blutderivate; diese Maßnahmen hindern die Mitgliedstaaten nicht daran, strengere Schutzmaßnahmen beizubehalten oder einzuführen».

Sofern mit einer Rechtsverordnung auf der Grundlage des § 30 Abs. 1 TFG ausschließlich EG-Recht umgesetzt wird, bedarf die Rechtsverordnung im Übrigen nicht der Zustimmung des Bundesrates ( $\$ 30$ Abs. 2 TFG) (zur Zulässigkeit der Umsetzung der EG-Blutrichtlinien 2002/98/EG und 2004/33/EG in nationales Recht durch Richtlinien der Bundesärztekammer nach $\S \S 12$ und 18 Transfusionsgesetz siehe [3]).

\section{Bislang keine Rechtsverordnung nach \$ 12 TFG}

Die Umsetzung der auf der EG-Blutrichtlinie 2002/98/EG beruhenden Durchführungsrichtlinien der Europäischen Kommission erfolgte unter anderem durch § 31 AMWHV (Ergänzende Regelungen für Blutspendeeinrichtungen) [13]. § 31 AMWHV wird jedoch erkennbar nicht auf $\S 12$ TFG gestützt. Es bleibt daher festzuhalten, dass bisher keine Rechtsverordnung auf der Grundlage der Ermächtigungsnorm des § 12 TFG erlassen wurde.

\section{Ermächtigungsgrundlage des $§ 12$ a TFG}

Die Ermächtigungsgrundlage des § 12a TFG für den Erlass der - untergesetzlichen - BlutRiLi wurde durch Artikel 3 Ziffer 4a des Gewebegesetzes in das TFG eingeführt und hat folgenden Wortlaut: 
«(1) Die Bundesärztekammer kann den allgemein anerkannten Stand der Erkenntnisse der medizinischen Wissenschaft und Technik zur Gewinnung von Blut und Blutbestandteilen ergänzend zu den Vorschriften der Rechtsverordnung nach \& $12 \mathrm{im}$ Einvernehmen mit der zuständigen Bundesoberbehörde in Richtlinien feststellen. (...)»

Zur amtlichen Begründung [14] heißt es:

«Die Bundesärztekammer wird ermächtigt, ergänzend zu den Anforderungen des Transfusionsgesetzes und der Verordnung nach § 12 TFG konkretisierende Richtlinien zum Stand der medizinischen Wissenschaft und Technik für die Gewinnung von Blut und Blutbestandteilen aufzustellen. Die Richtlinien bedürfen der Anhörung durch die einschlägigen Fachkreise und der zuständigen Behörden von Bund und Ländern und können nur im Einvernehmen mit der zuständigen Bundesoberbehörde erlassen werden, um die sachgerechte Abgleichung mit den Standards der Verordnung nach $\S 12 \mathrm{zu}$ gewährleisten und mögliche Diskrepanzen zu vermeiden. Es wird darüber hinaus an dem bisherigen Recht festgehalten, wonach die Richtlinien von der zuständigen Bundesoberbehörde im Bundesanzeiger bekannt zu machen sind. Dies soll die Transparenz und Akzeptanz der Richtlinien in den Fachkreisen erhöhen. Durch die Einführung dieser Richtlinienkompetenz wird im Übrigen erreicht, dass neben der Verordnung nach § 12 TFG ein Regelwerk geschaffen werden kann, das den Stand der medizinischen Wissenschaft und Technik flexibel wiedergibt."

\section{§ 12a TFG als Rechtsgrundlage für die neuen BlutRiLi?}

Aufgrund des Wortlauts des $\S 12 \mathrm{a}$ Abs. 1 TFG und der amtlichen Begründungen zu $\S \S 12,12 \mathrm{a}$ TFG ist zweifelhaft, ob - wie in Kapitel 1.1 BlutRiLi dargestellt - $\S 12 \mathrm{a}$ Abs. 1 TFG zum gegenwärtigen Zeitpunkt tatsächlich als Rechtsgrundlage für die BlutRiLi der Bundesärztekammer herangezogen werden kann.

\section{Contra}

Hiergegen spricht der ausdrückliche Wortlaut des § 12a Abs. 1 TFG, wonach die Bundesärztekammer den allgemein anerkannten Stand der Erkenntnisse der medizinischen Wissenschaft und Technik zur Gewinnung von Blut und Blutbestandteilen lediglich ergänzend $\mathrm{zu}$ den Vorschriften der Rechtsverordnung nach $\S 12$ TFG im Einvernehmen mit der zuständigen Bundesoberbehörde in Richtlinien feststellen kann. Es besteht also unbezweifelbar ein Primat der Rechtsverordnung. Die Bundesärztekammer wird also kraft Gesetzes gerade nicht generell zum Erlass von Richtlinien zur Feststellung (nicht: Festlegung) des allgemein anerkannten Stands der medizinischen Wissenschaft und Technik ermächtigt, sondern nur dann, wenn eine auf $\S 12$ TFG gestützte Rechtsverordnung existiert

Nach der amtlichen Begründung zu $§ 12$ TFG tritt die Verordnungsermächtigung zugunsten des Bundesministeriums für Gesundheit an die Stelle der ursprünglichen Richtlinienkompetenz der Bundesärztekammer und der zuständigen Bundesoberbehörde zur alleinigen Feststellung des allgemein anerkannten Stands der medizinischen Wissenschaft und Technik. Die Bundesärztekammer kann lediglich «weitere oder konkretisierende Standards» festlegen, sofern dies erforderlich sein sollte. Sie soll daher nach dem eindeutigen gesetzgeberischen Willen nicht generell, und nicht etwa subsidiär oder gar verordnungsvertretend, tätig werden, sondern nur «ergänzend»bzw. «neben einer Verordnung nach $\S 12$ TFG», wie sich auch der amtlichen Begründung zu $§ 12 \mathrm{a}$ TFG entnehmen lässt.

Pro

Für die Auffassung, dass die Bundessärztekammer auch ohne Vorliegen einer nach § 12 TFG erlassenen Rechtsverordnung in Richtlinien den allgemein anerkannten Stand der Erkenntnisse der medizinischen Wissenschaft und Technik zur Gewinnung von Blut und Blutbestandteilen festlegen kann - oder vielmehr können muss -, könnten rein praktische Erwägungen sprechen. Die BlutRiLi der Bundesärztekammer haben sich in der Praxis durchaus bewährt. Es ist besteht kein Zweifel daran, dass die BlutRiLi - auch wenn ihnen nach § 12a Abs. 2 TFG «nur» eine Vermutungsregelung zukommt ( $\$ 12$ a Abs. 2 TFG lautet: «Die Einhaltung des Standes der Erkenntnisse der medizinischen Wissenschaft und Technik wird vermutet, wenn die Richtlinien der Bundesärztekammer nach Absatz 1 beachtet worden sind.») - einen hilfreichen Leitfaden für alle Ärzte darstellen, die unter anderem mit dem Gewinnen, Herstellen, Lagern, Abgeben oder Inverkehrbringen von Blut, Blutbestandteilen oder Blutprodukten sowie der Durchführung von blutgruppenserologischen und weiteren immunhämatologischen Untersuchungen befasst sind. Nur dann, wenn sich tatsächlich ergeben sollte, dass in der Praxis Mängel bestehen, auf die die zuständigen Fachkreise nicht reagieren oder die durch die zuständigen Überwachungsbehörden nicht beseitigt werden oder nicht beseitigt werden können, besteht überhaupt das Bedürfnis, dass das BMG eine Verordnung, gestützt auf $\$ 12$ TFG, erlässt. Die BlutRiLi tragen entscheidend dazu bei, dass ein solcher Zustand gar nicht erst entsteht bzw. eventuell bestehende Mängel in zufriedenstellender Weise beseitigt werden können.

Ein weiteres Argument, ohne Vorliegen einer Rechtsverordnung zu Recht die BlutRiLi zu erlassen, könnte möglicherweise $\S 20$ TFG entnommen werden. Diese Rechtsverordnungsermächtigung, die die Rückverfolgbarkeit betrifft, lautet:

«Das Bundesministerium für Gesundheit wird ermächtigt, nach Anhörung von Sachverständigen eine Rechtsverordnung mit Zustimmung des Bundesrates zur Regelung der Einzelheiten des Verfahrens der Rückverfolgung zu erlassen, sofern dies zur Abwehr von Gefahren für die Gesundheit von Menschen oder zur 
Risikovorsorge erforderlich ist. Mit der Verordnung können insbesondere Regelungen zu einer gesicherten Erkennung des Infektionsstatus der spendenden und der zu behandelnden Personen, zur Dokumentation und zur Übermittlung von Daten zu Zwecken der Rückverfolgung, zum Zeitraum der Rückverfolgung sowie zu Sperrung und Lagerung von Blutprodukten erlassen werden.»

Diesbezüglich heißt es in der Literatur [11, § 20 Rdn. 3] wie folgt:

«Die Regelungen (gemeint: gesetzliche Regelungen und eine Reihe von Voten des Arbeitskreises Blut) haben sich bewährt. Unter diesen Umständen hat der Verordnungsgeber bisher darauf verzichtet, von der Ermächtigung des $\S 20$ Gebrauch zu machen. Sollten die Regeln des AK Blut-Votums 24 nicht ausreichen, nicht beachtet oder nicht dem Stand der wissenschaftlichen Erkenntnisse angepasst werden, wird der Erlass der Verordnung in Betracht gezogen werden müssen. Solange aber die Regeln der Fachkreise ihren Zweck erfüllen, sollen sie maßgeblich bleiben, zumal sie das flexiblere und rascher zu novellierende Instrument sind.»

Eine solche Parallele kann allerdings allenfalls zu der Vorschrift des $§ 12$ TFG gezogen werden. Sie würde nur erklären, warum der Verordnungsgeber bislang keinen Handlungsbedarf sieht. Eine dem $\S 12$ a TFG als Richtlinienermächtigung zugunsten der Bundesärztekammer vergleichbare Vorschrift findet sich im vierten Abschnitt des TFG bezüglich der Rückverfolgbarkeit gerade nicht.
Außerdem ist zweifelhaft, ob angenommen werden kann, dass aus Sicht des Verordnungsgebers bislang tatsächlich kein Handlungsbedarf besteht. Die in der Verordnungsermächtigungsnorm des § 12 TFG genannten Regelungsgegenstände (siehe oben «Wortlaut und amtliche Begründung» zu § 12 TFG) sind im Wesentlichen deckungsgleich mit denen der BlutRiLi. Soweit die Gewinnung von Blut betroffen ist, dienen die in der BlutRiLi behandelten Gesichtspunkte dem Schutze der spendenden Person und damit der Risikovorsorge, wie dies in $\S 12$ Abs. 1 TFG vorausgesetzt wird. Nimmt die Bundesärztekammer diese Sicherheitsaspekte wahr, steht damit - wenn auch nur inzident - fest, dass es insoweit tatsächlich erforderlich ist, aus Gründen der Gefahrenabwehr und/oder Risikovorsorge zu handeln. Dann aber müsste zunächst der Verordnungsgeber nach der derzeitigen Fassung der $\S \S 12$ und 12a TFG tätig werden. Es kann aus rechtsstaatlichen Gründen nicht im Belieben einer untergesetzlichen Körperschaft (Bundesärztekammer) stehen, einen Regelungsgegenstand zu usurpieren, der de lege lata dem Staat in Form einer Rechtsverordnung vorbehalten ist.

\section{Ergebnis}

Es kann festgehalten werden, dass jedenfalls rechtliche Zweifel bestehen, ob die BlutRiLi, soweit sie die Gewinnung von Blut und Blutbestandteilen betreffen, in rechtmäßiger Weise gestützt auf $\$ 12 \mathrm{a}$ TFG erlassen werden konnten. Dagegen besteht kein Zweifel daran, dass hinsichtlich der Anwendung von Blutprodukten die Bundesärztekammer zum Richtlinienerlass auf der Grundlage des $\S 18$ TFG gesetzlich ermächtigt ist.

\section{Literatur}

1 Gesetz über Qualität und Sicherheit von menschlichen Geweben und Zellen vom 20. Juli 2007 (BGBl. I S. 1574)

2 Gesetz zur Regelung des Transfusionswesens (Transfusionsgesetz - TFG) vom 1. Juli 1998 (BGB1. I S. 1752), geändert durch erstes Gesetz zur Änderung des Transfusionsgesetzes und arzneimittelrechtlicher Vorschriften vom 10. Februar 2005 (BGBl. I S. 234).

3 Hasskarl H: Zur Zulässigkeit der Umsetzung der EG-Blutrichtlinien 2002/98/EG und 2004/33/EG in nationales Recht durch Richtlinien der Bundesärztekammer nach $\$ \S 12$ und 18 Transfusionsgesetz. Transfus Med Hemother, 2005:32:34-41.

4 BT-Drucksache 16/5443 vom 23. Mai 2007, S. 80 f. der elektronischen Vorab-Fassung.

5 Gesetz zur Regelung des Transfusionswesens (Transfusionsgesetz - TFG) vom 28. August 2007 (BGBl. I S. 2169), zuletzt geändert durch Gesetz vom 17. Juli 2009 (BGBl. I S. 1990).

6 BT-Drucksache 16/3146 vom 25. Oktober 2006, S. 43.
7 Richtlinie 2004/33/EG der Kommission vom 22. März 2004 zur Durchführung der Richtlinie 2002/98/EG des Europäischen Parlaments und des Rates hinsichtlich bestimmter technischer Anforderungen für Blut und Blutbestandteile (ABl. EU Nr. L 91/25 vom 30. März 2004).

8 Richtlinie 2005/61/EG der Kommission vom 30. September 2005 zur Durchführung der Richtlinie 2002/98/EG des Europäischen Parlaments und des Rates in Bezug auf die Anforderungen an die Rückverfolgbarkeit und die Meldung ernster Zwischenfälle und erster unerwünschter Reaktionen (AB1. EU Nr. L 256/325 vom 1. Oktober 2005).

9 Richtlinie 2005/62/EG der Kommission vom 30. September 2005 zur Durchführung der Richtlinie 2002/98/EG des Europäischen Parlaments und des Rates in Bezug auf gemeinschaftliche Standards und Spezifikationen für ein Qualitätssystem für Blutspendeeinrichtungen (AB1. EU Nr. L 256/41 vom 1. Oktober 2005).
10 Richtlinie 2002/98/EG des Europäischen Parlaments und des Rates vom 27. Januar 2003 zur Festlegung von Qualitäts- und Sicherheitsstandards für die Gewinnung, Testung, Verarbeitung, Lagerung und Verteilung von menschlichem Blut und Blutbestandteilen und zur Änderung der Richtlinie 2001/83/EG (ABl. EU Nr. L 33/30 vom 8. Februar 2003).

11 Von Auer F, Seitz R: Gesetz zur Regelung des Transfusionswesens (Transfusionsgesetz), Kommentar und Vorschriftensammlung, 14. Lieferung, Stand: Oktober 2009.

12 Vertrag über die Arbeitsweise der Europäischen Union (ABl. EU Nr. C 83/47 vom 30. März 2010).

13 Verordnung über die Anwendung der Guten Herstellungspraxis bei der Herstellung von Arzneimitteln und Wirkstoffen und über die Anwendung der Guten fachlichen Praxis bei der Herstellung von Produkten menschlicher Herkunft (Arzneimittelund Wirkstoffherstellungsverordnung - AMWHV) vom 3. November 2006 (BGBl. I S. 2523), zuletzt geändert durch Verordnung vom 26. März 2008 (BGBl. I S. 521).

14 BT-Drucksache 16/5443 vom 23. Mai 2007, S. 108. 